\title{
Disability and quality of life after surgery for cancer
}

\begin{abstract}
Purpose: We aimed to assess the prevalence of disability and health-related quality of life (HRQoL), as well as associated risk factors among patients operated for gastrointestinal or thoracic cancer.

Methods: A retrospective study was performed on 97 survivor patients underwent curative surgery for gastrointestinal or thoracic cancer. Subjects were contacted by phone call between 6-12 months after surgery. Completely dependent patients in any item of modified Katz index were defined as disabled. Severe impairment in HRQoL was defined as $\leq 50$ points in the EuroQoL-5D-3L-visual analogue scale.

Results: The median age of 63.0 years (IQR 56.0-71.0years). Gastrointestinal and thoracic surgery was performed in $67(69.1 \%)$ and 30 patients $(30.9 \%)$, respectively. Thirty-eight $(39.2 \%)$ participants develop a postoperative major complication. The disability and severe impairment in HRQoL prevalence was $17.5 \%$ and $19.5 \%$, respectively. Advanced cancer $(52.9 \%$ vs. $25.0 \%$; p=0.046), ASA class $\geq 3$ (52.9\% vs. $22.5 \% ; \mathrm{p}=0.030)$ and postoperative major complications $(58.8 \%$ vs. $23.8 \%$; $=0.010)$, while advanced cancer $(52.6 \%$ vs. $24.4 \% ; \mathrm{p}=0.033)$, postoperative major complications $(63.2 \%$ vs. $25.6 \% ; p=0.004)$ and disability $(73.7 \%$ vs. $24.4 \% ; p=0.0001)$ were the risk factor for severe impairment in HRQoL.

Conclusions: Disability and severe impairment in HRQoL are uncommon in patients operated for thoracic or gastrointestinal cancer. However, some risk factors such as advanced cancer, ASA class $\geq 3$ and postoperative major complications could aid in establishing a directed rehabilitation program for preventing disability and improve HRQoL in this population.
\end{abstract}

Keywords: cancer, surgery, quality of life, activities of daily living, disability, postoperative complications
Volume I Issue 4 - 2017

\author{
Frank Daniel Martos Benítez, Andrés Soto \\ García, Anarelys Gutiérrez Noyola, Iraida \\ González Martínez, llionanys Betancour \\ Plaza, Francy Noheli Meza Pedríquez \\ Department of Intensive Care, Institute of Oncology and \\ Radiobiology, Cuba
}

Correspondence: Frank Daniel Martos Benítez, Department of Intensive Care, Institute of Oncology and Radiobiology, Fuentes street No. 367A, Guanabacoa, Havana, Cuba, Tel +53 53925706,Email fdmartos@infomed.sld.cu

Received: August 28, 2017 | Published: September 26, 2017
Abbreviations: HRQoL, health related quality of life; ADL, activities of daily living; QoL, quality of life; IOR, institute of oncology and radiobiology; ACCI, adjusted charlson comorbidity index; ASA, american society of anaesthesiology; APCHE, acute physiology and chronic health evaluation; VAS, visual analogue scale; IQR, interquartile range; PS, performance status

\section{Introduction}

Cancer is one of first cause of death around the world and Cuba. Surgery is one of most important therapeutic tool for its control, either for curative or palliative purpose. Although surgical intervention can be a life-saviour strategy for these patients, several complications could be present in postoperative period, ${ }^{2}$ as well as limitations for activities of daily living (ADL) and derangement in quality of life (QoL). ${ }^{3}$ Postoperative recovery is a dynamic process where biological, physiological, functional and psychological components play a pivotal role. A rise in physical independence and gradual return to ADL are global indicator of functional recovery after surgery. For most patients, the evaluation of ADL is only performed during their hospital stay. Traditionally, patients should probe their capacity to walking, eating, bathing and dressing without help. ${ }^{4}$

Elderly patients are a special increasing subgroup. ${ }^{5}$ Because of the ageing-related characteristics, this population has a decreased functional capacity even before hospital admission. ${ }^{6}$ According to literature reports, more than $10 \%$ of elderly patients develop a severe postoperative disability. ${ }^{7}$ Postoperative evaluations of functional capacity and health-related QoL (HRQoL) have been become as an important component of long-term surgical outcomes, particularly in the elderly population. ${ }^{8-11}$

The problem is more serious in the case of cancer patients because a higher prevalence of malignancies among the elderly subgroup. ${ }^{12,13}$ Frequently, surgical indication for elderly patients with comorbidities is based on subjective judgement according to the personal experience of surgeon. In addition, a negative attitude regarding elderly patients can be see among some physicians, which lead to patients with acceptable functional status could be not surgically treated as usually is indicated for younger patients. Furthermore, radical surgical interventions are neither performed in elderly population. This is significant because currently the primary tumour and regional lymphatic node can only be removed by radical intervention with surgical margin, which guarantees a higher probability of cure. ${ }^{14,15}$ This study was aimed to assess the prevalence of disability and healthrelated quality of life, as well as related risk factors among patients operated for gastrointestinal or thoracic cancer.

\section{Methods}

\section{Design and setting}

This retrospective study was conducted in the oncological ICU (OICU) of the Institute of Oncology and Radiobiology (IOR). This is a 220-bed, university-affiliated, tertiary care referral centre for cancer patients in Havana, Cuba. The OICU has 12 beds and provides care for about 400 surgical cancer patients per year. The current study was 
conducted in accordance with the Declaration of Helsinki, and it was approved by the Scientific Council and the Ethics Committee for Scientific Research of the IOR (September 2014).

\section{Participants}

Using the prospective database of the OICU, patients underwent thoracic (lung, mediastinum) or gastrointestinal (oesophagus, stomach, small intestine, pancreas, liver, biliary, colon or rectum) curative surgery for cancer were selected from July 2013 to July 2014. Of these patients, those operated six to 12 month before, discharged alive from the hospital, and with residence in Havana were included (Figure 1).

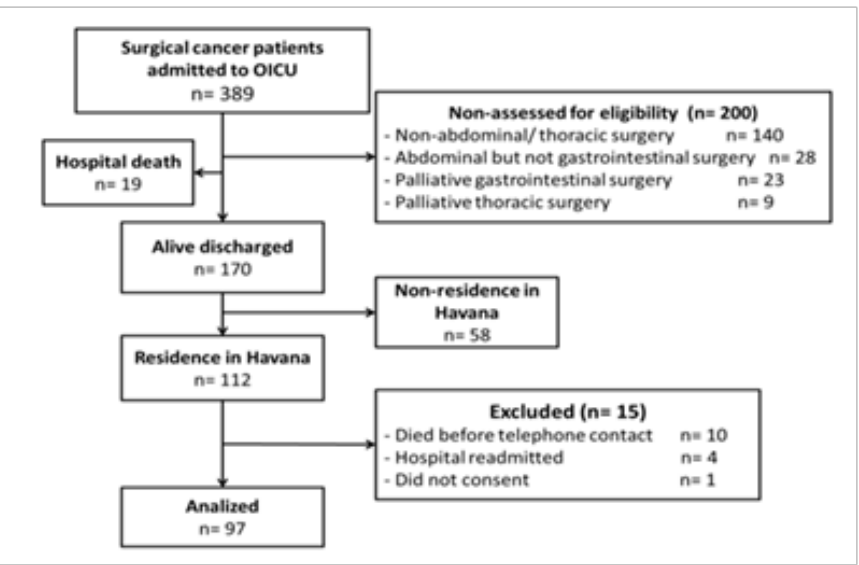

Figure I Flow diagram of study participants. OICU, oncological intensive care unit.

Telephone numbers were searched in hospital records and all included subjects were called (November 2014-February 2015). The following patients were excluded:

i. Who died before telephone contact;

ii. Readmitted after initial hospital discharge; and

iii. Not consent to be included in the study. The flow diagram of participants is depicted in Figure 1.

\section{Data collection and outcomes}

The following variables on OICU admission were extracted from the medical records: age, sex, Age-Adjusted Charlson Comorbidity Index (ACCI), cancer stage, surgical location, emergency surgery, American Society of Anaesthesiology (ASA) class, intraoperative complication, surgical time, Acute Physiology and Chronic Health Evaluation (APACHE) II score, postoperative major complications (unplanned re-intervention and/or organ dysfunction), ${ }^{16}$ and length of OICU and hospital stay.

A modified Katz index scale was applied to measure the ADL

Table I General characteristics of participants in our study, which included the following six items: dressing, feeding, transferring (getting in/out of bed), walking (walking around inside), bathing, and toileting. Each item had three response choices: "completely independent", "needing some help", and "completely dependent". If any answer was "completely dependent", participants were defined as disabled; otherwise the participants were categorized as nondisabled. ${ }^{17}$

HRQoL were assessed using the EuroQoL-5D-3L, which is a brief self-reported generic measure of current health that consists of five dimensions (Mobility, Self-Care, Usual Activities, Pain/Discomfort, and Anxiety/Depression), each with three levels of functioning (no problems, some problems, and unable to/extreme problems). In addition, the EuroQoL-5D-3L includes a visual analogue scale (VAS) where own health "today" is rated on a scale from 0 (worst imaginable health) to 100 (best imaginable health). Severe impairment in HRQoL was defined as $\leq 50$ points in VAS. Both the modified Katz index ${ }^{3,17-21}$ and EuroQoL-5D-3L $\mathrm{L}^{22-30}$ has been widely used in different clinical setting, including surgical cancer patients.

\section{Statistical analysis}

Categorical variables are shown as count with percentage, whereas continuous variables are represented as median with $25^{\text {th }}$ $75^{\text {th }}$ interquartile range (IQR). Between-group comparisons were performed using the chi-square $\left(\chi^{2}\right)$ test or Fisher's exact test based on which test was more suitable for qualitative variables. Because of lack of normality, the Mann-Whitney $U$ test was used for numerical variables. Statistical test with a two tailed $p$-value $\leq 0.05$ was considered as significant. Data were analysed using IBM ${ }^{\circledR}$ SPSS $^{\circledR}$ Statistics 23.0 (IBM, Chicago, IL, USA).

\section{Results}

\section{Characteristics of study population}

Ninety-seven patients were analysed with a median age of 63.0 years (IQR 56.0-71.0years). Comorbidities were measured by the ACCI score (median 3.0 points (IQR 2.0-5.0 points); a score $\geq 2$ points was observed in $86.6 \%$ of subjects. Gastrointestinal and thoracic surgery was performed in $67(69.1 \%$ ) and 30 patients $(30.9 \%)$, respectively. The most common gastrointestinal surgery was colorectal $(55.2 \%)$, whereas pulmonary resection $(73.3 \%)$ was the predominant thoracic surgery. The emergency surgery was carried out in $8.2 \%$ of patients. Intraoperative complications were observed in $5.2 \%$ (Table 1). The median APACHE II score was 10.0 points (IQR $8.0-12.0$ points) with $11.1 \%$ (RIQ $8.1-14.6 \%$ ) as median estimated probability of death. The median time of OICU stay after surgery and overall time of hospitalization was 3.0 days (IQR 2.0-5.0 days) and 8.0 days (IQR 7.0-11.0 days), respectively. Because of the curative intention of the surgery, all patients received oncological therapy after surgery such as chemotherapy $(52,6 \%)$, radiotherapy $(30,9 \%)$ or both $(16,5 \%)$.

\begin{tabular}{ll}
\hline Characteristic & $\mathrm{N}=97$ \\
\hline Age, years (IQR) & $63(56-71)$ \\
$\geq 60$ years, $\mathrm{n}(\%)$ & $61(62.9)$ \\
$\geq 80$ years, $\mathrm{n}(\%)$ & $4(4.1)$ \\
\hline
\end{tabular}




\begin{tabular}{ll} 
Table Continued.... & \\
\hline Characteristic & $\mathbf{N}=97$ \\
\hline Gender, n(\%) & \\
Male & $40(4 I .2)$ \\
Female & $57(58.8)$ \\
Age-adjusted charlson comorbidity index, points (IQR) & $\mathbf{3 ( 2 - 5 )}$ \\
Advanced cancer (stage IIIb-IV), n(\%) & $28(28,9)$ \\
ASA class $\geq 3$, n(\%) & $39(40.2)$ \\
Surgical Location, n(\%) & \\
Gastrointestinal surgery & $67(69.1)$ \\
Oesophagus & $20(29.9)$ \\
Stomach & $4(13.3)$ \\
Colorectal & $37(55.2)$ \\
Hepato-bilily-pancreatic & $3(4.5)$ \\
Others & $7(10.4)$ \\
Thoracic surgery & $30(30.9)$ \\
Pulmonary resection & $22(73.3)$ \\
Mediastinum & $4(13.3)$ \\
Emergency surgery, n(\%) & $\mathbf{8 ( 8 . 2 )}$ \\
Intraoperative complications, n(\%) & $\mathbf{5 ( 5 . 2 )}$ \\
Surgical time, minutes(IQR) & $\mathbf{2 3 4 ( I 9 6 - 3 0 5 )}$ \\
APACHE II score, points(IQR) & $\mathbf{1 0 ( 8 - 1 2 )}$ \\
Length of OICU stay, days(IQR) & $\mathbf{3 . 0 ( 2 . 0 - 5 . 0 )}$ \\
Length of hospital stay, days(IQR) & $\mathbf{8 . 0 ( 7 . 0 - 1 I . 0 )}$ \\
\hline
\end{tabular}

APACHE, acute physiology and chronic health evaluation;ASA, American society of anaesthesiology; IQR, interquartile ranges

\section{Postoperative complications}

At least one postoperative major complication occurred across 38 participants (39.2\%). Medical and surgical postoperative complications were found in $31(32.0 \%)$ and $15(15.5 \%)$ patients, respectively. Eight subjects $(8.2 \%)$ developed both medical and surgical complications. A total of 45 complications were diagnosed. Of these, the most common complications were delirium (22.2\%) and pneumonia $(22.2 \%)$, followed by atelectasis $(13.3 \%)$ and anastomotic leak $(13.3 \%)$. Other postoperative complications were surgical wound infection, postoperative haemorrhage, acute heart failure, intestinal perforation/ obstruction, persistent air-pulmonary leak and broncopleural fistula (Figure 2). Infectious complications were found in 20 patients (44.4\%) and four (8.9\%) develop septic shock.

Medical postoperative complications were significantly more frequent than surgical postoperative complications (68.9\% vs. $31.1 \%$; $\mathrm{p}<0.0001)$. Compared with patients underwent thoracic surgery, those experienced gastrointestinal surgery had a higher complications rate ( $56.7 \%$ vs. $31.3 \% ; \mathrm{p}=0.033)$. The overall median length of OICU and hospital stay was 3.0 days (IQR 2.0-5.0 days) and 9.0 days (IQR 7.0-14.0 days), respectively. Thoracic and gastrointestinal surgical patients had a median length of hospital stay of 8.0 days (IQR 6.8-12.3 days) and 10.0 days (IQR 8.0-15.0 days), respectively. Furthermore, a significant statistically difference was not observed between groups $(\mathrm{p}=0,214)$.

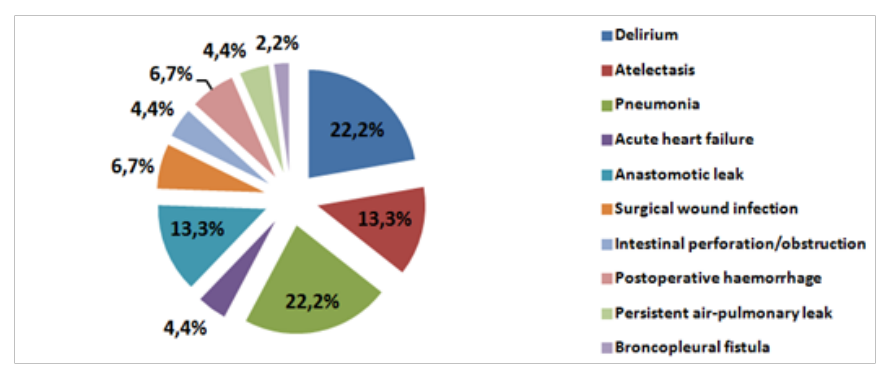

Figure 2 Postoperative major complications.

\section{Assessment of disability}

The overall disability prevalence was $17.5 \%$. The prevalence of disability was highest for "transferring" $(6.2 \%)$, followed by "walking" $(5.2 \%)$ and "bathing" (5.2\%) (Figure 3). There was no difference between patients underwent gastrointestinal surgery and those experienced thoracic surgery regarding disability prevalence $(68.8 \%$ vs. $70.6 \%$; $\mathrm{p}=1.000)$. Advanced cancer $(52.9 \%$ vs. $25.0 \%$; $=0.046)$, 
ASA class $\geq 3(52.9 \%$ vs. $22.5 \%$; $=0.030)$ and postoperative major complications $(58.8 \%$ vs. $23.8 \% ; \mathrm{p}=0.010)$ rates were significantly higher in patients with disability than those observed in patients without disability (Table 2)

Table 2 Factors associated with limitations for activities of daily living

\begin{tabular}{|c|c|c|c|}
\hline Characteristic & Disabled N=I7 & Nondisabled $\mathbf{N}=80$ & $\mathbf{p}$ \\
\hline Age, years (IQR) & $63.4(54.2-72,6)$ & $61.6(52.0-71.2)$ & 0.407 \\
\hline Gender (male), n (\%) & $8(47.1)$ & $35(43.8)$ & 0.985 \\
\hline Advanced Cancer (stage IIIb-IV), n (\%) & $9(52.9)$ & $20(25.0)$ & 0.046 \\
\hline ASA class $\geq 3, n$ (\%) & $9(52.9)$ & $18(22.5)$ & 0.03 \\
\hline Surgical location, n (\%) & & & 0.855 \\
\hline Gastrointestinal surgery & $10(58.8)$ & $48(60.0)$ & \\
\hline Thoracic surgery & $7(4 \mid .2)$ & $32(40.0)$ & \\
\hline Emergency surgery, n (\%) & $2(11.8)$ & $5(6.3)$ & 0.71 \\
\hline Intraoperative complications, $\mathrm{n}$ (\%) & $5(29.4)$ & $16(20.0)$ & 0.576 \\
\hline Surgical time, minutes (IQR) & $229(191-299)$ & $225(189-297)$ & 0.681 \\
\hline APACHE II score, points (IQR) & II (8-I3) & $10(8-12)$ & 1 \\
\hline Postoperative major complication, $n(\%)$ & $10(58.8)$ & 19(23.8) & 0.01 \\
\hline
\end{tabular}

$A D L$, activities of daily living; IQR, interquartile ranges

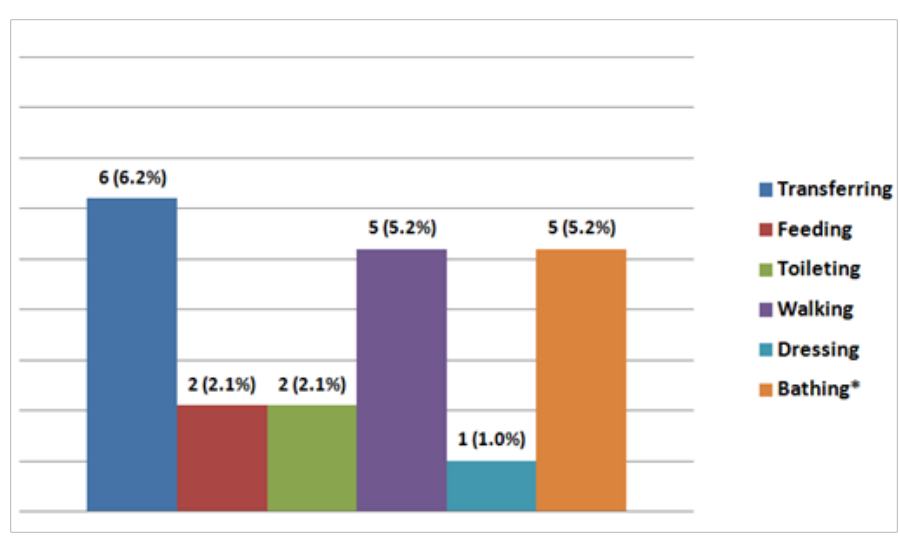

Figure 3 Activities of daily living according to modified katz index.

*:A same patient could have more than one limitation.

\section{Health-related quality of life}

The most affected dimension in the EuroQoL-5D-3L was "mobility" (51.5\% with some or extreme problems), followed by "pain/ discomfort" ( $45.4 \%$ with some or extreme problems) and "anxiety/ depression" (39.2\% with some or extreme problems). However, dimension "usual activities" showed the highest rate of extreme problems. The lowest affected dimension was "self-care" because $72.2 \%$ of patients had not problems (Table 3 ). The median VAS was 73 points (IQR 58.0-81.0 points). A VAS less than or equal to 50 and 25 points was found in $19.6 \%$ and $10.3 \%$ of patients, respectively. There was no statistical difference regarding VAS between gastrointestinal surgical patients and those underwent thoracic surgery (median 73.5 points [IQR 63.3-78.8 points] vs. median 73.0 points [IQR 56.081.0 points]; $\mathrm{p}=0.913$ ). Factors associated with severe impairment in health-related quality of life were advanced cancer (52.6\% vs. $24.4 \%$; $\mathrm{p}=0.033)$, postoperative major complications $(63.2 \%$ vs. $25.6 \%$; $\mathrm{p}=0.004)$ and disability $(73.7 \%$ vs. $24.4 \%$; $=0.0001)$ (Table 4$)$.

Table 3 Health-related quality of life according to the EuroQoL-5D-3L

\begin{tabular}{llll}
\hline \multirow{2}{*}{ Dimension } & \multicolumn{2}{l}{ Level } & \\
\cline { 2 - 4 } & No problems & Some problems & Extreme problems \\
\hline Mobility & $47(48.5)$ & $39(40.2)$ & $1 \mathrm{I}(\mathrm{II.3)}$ \\
Self-care & $70(72.2)$ & $17(17.5)$ & $10(10.3)$ \\
Usual Activities & $57(58.8)$ & $21(21.6)$ & $19(19.6)$ \\
Pain/Discomfort & $53(54.6)$ & $34(35.1)$ & $10(10.3)$ \\
Anxiety/Depression & $59(60.8)$ & $26(26.8)$ & $12(12.4)$ \\
\hline
\end{tabular}


Table 4 Factors associated with impairment in health-related quality of life

\begin{tabular}{|c|c|c|c|}
\hline Characteristic & $\begin{array}{l}\text { Severe impairment in HRQoL } \\
N=19\end{array}$ & $\begin{array}{l}\text { No Severe impairment in HRQoL } \\
\mathrm{N}=78\end{array}$ & $\mathbf{p}$ \\
\hline Age, years (IQR) & $64.2(55.2-73.5)$ & $62.3(52.5-70.1)$ & 0.53 \\
\hline Gender (male), n (\%) & $9(47.4)$ & $34(43.6)$ & 0.968 \\
\hline Advanced Cancer (stage IIIb-IV), n(\%) & $10(52.6)$ & $19(24.4)$ & 0.033 \\
\hline ASA class $\geq 3, n(\%)$ & $9(47.4)$ & $19(24.4)$ & 0.09 \\
\hline Surgical location, n(\%) & & & 0.808 \\
\hline Gastrointestinal surgery & II (57.9) & $45(57.7)$ & \\
\hline Thoracic surgery & $8(42.1)$ & $33(42.3)$ & \\
\hline Emergency surgery, $n(\%)$ & $3(15.8)$ & $5(6.4)$ & 0.373 \\
\hline Intraoperative complications, n(\%) & $9(47.4)$ & $18(23.1)$ & 0.067 \\
\hline Surgical time, minutes (IQR) & $233(190-301)$ & $226(190-299)$ & 0.467 \\
\hline APACHE II Score, points (IQR) & $12(9-\mid 4)$ & II (8-13) & 0.054 \\
\hline Postoperative major complication, n(\%) & $12(63.2)$ & $20(25.6)$ & 0.004 \\
\hline Disability, n(\%) & |4(73.7) & $19(24.4)$ & 0.0001 \\
\hline
\end{tabular}

IQR, interquartile ranges; QoL, quality of life

\section{Discussion}

The health status before surgery, as well as postoperative complications can affect the well-being of patients undergoing surgical intervention for cancer at short, medium and long term. Thus medical, social, and functional points of view are important to anticipate the expected postoperative functional status and care needs prior to surgery, and to inform patients and their families.

Disability is a frequently observed condition at hospital discharge with a prevalence of almost $30 \%{ }^{31}$ Additionally, changes in the functional status of the cancer patients are common, which could affect the prognosis; consequently, applying the assessment tools is important to assist clinical oncologist. Thus disability has been assessed in several studies of cancer patients..$^{3,8}$ Recently, Khoei et al. ${ }^{32}$ proved that the Katz index is a reliable instrument for oncologic patients using the Physical Function subscale of SF 36 as criterion of validity (Cronbach's alpha 0.923). ${ }^{32}$

Elderly patients were the most frequent population in our study. This is not surprising because the elderly population is at higher risk of cancer. ${ }^{33}$ It is well established that declines in physical function are an inevitable part of the aging process and are markers of disability risk. ${ }^{6}$ Once the elderly become disabled, their physical health declines along with their mental health, their quality of life deteriorates rapidly and they require long-term care. ${ }^{34} \mathrm{~A}$ multicentre prospective study of 639 institutionalized elderly patients with and without cancer showed a disability prevalence of $33 \%{ }^{35}$ This study showed that $17.5 \%$ of patients experienced disability after abdominal or thoracic surgery for cancer. Furthermore, advanced cancer, ASA class $\geq 3$ and postoperative major complications were the risk factors associated with disability.

Although the functional status such as ADL and QoL are important for outcomes assessment in postoperative cancer patients, there have been only a limited number of reports on the natural course of recovery of functional independence. In patients operated for gastric or colorectal cancer, Amemiya et al. ${ }^{3}$ observed a disability rate of $24 \%$. The surgical risk predictive scales such as POSSUM and E-PASS were the associated risk factors. ${ }^{3}$ We observed a disability rate of $17 \%$. These differences with our results appear to derive from differences in the composition of the studied participants. For example, in our study only postoperative patients were included.

In patients receiving lobectomy for non-small cell lung cancer, Kawaguchi et al. observed an incidence of $18.2 \%$ for the decline in postoperative performance status (PS), assessed by the Eastern Cooperative Oncology Group PS. Risk factors for decreasing PS after surgery were history of cardiac ischemia $(\mathrm{p}=0.001)$ and squamous cell carcinoma $(\mathrm{p}=0.015)$. Authors also found a higher overall 5 -year survival rate for patients with postoperative PS $<$ preoperative PS than those for patients with postoperative PS=reoperative PS. ${ }^{36}$

Saraiva et al. ${ }^{37}$ found that a better functional status, based on METs, was associated with reduced odds of postoperative complications in multivariate analysis (odds ratio $0.11 ; 95 \%$ CI $0.02-0.85 ; \mathrm{p}=0.034$ ) ${ }^{37}$ Conversely, we observed that postoperative major complications were a risk factor for limitations in ADL. Thus the relationship between postoperative complications and physical performance is a dynamic process with two-way effects. Hence, impairment in physical performance and limitations for basic ADL are important risk factors for subsequent disability and erosion of independent living, institutionalization, compromised quality of life and death, especially in older people operated for cancer. The need to develop practical and accessible approaches for the prevention of decline in functional performance can be considered a key public health priority.

Almost $20 \%$ of patients experienced a severe impairment in HRQoL in our study. Additionally, advanced cancer, postoperative major complications and disability were the associated risk factors. Regarding HRQoL, in patients operated for gastric cancer, Lim et 
al. ${ }^{11}$ observed that postoperative nutritional status has a negative effect on HRQoL, especially regarding global health, physical functions, fatigue, pain, appetite, reflux and anxiety. Consequently, a nutritional intervention for patients undergoing upper gastrointestinal tract surgery for cancer is mandatory, particularly for malnourished patients or at risk.

Brown et al. studied the effects of postoperative complications on HRQoL of 614 patients underwent colorectal surgery for cancer. The postoperative complication rate was $35 \%$. The most common dimensions affected in HRQoL were mobility, self-care and pain/ discomfort. ${ }^{8}$ These results coincide with our find; thus postoperative complications should be taken into account to assess the HRQoL after surgery. Postoperative complications are associated with an ADL declined. ${ }^{3}$ We found that disability was related with impairment in HRQoL. Consequently, postoperative complications, through the limitations for ADL, could explain its negative effect on HRQoL.

Physical activity interventions and the incorporation of physical activity into a healthy lifestyle represent at least one behavioural modality for possibly attenuating the effects of the surgical damage on functional limitations and disability and the attendant declines in quality of life. ${ }^{38}$ One potential way to understand the relationship between physical activity, functional limitations and QoL may be through the pathways of self-efficacy and physical function performance. That is, more active individuals are likely to be more efficacious, which should lead to better physical functional performance, fewer limitations and better well-being.

Several systematic reviews on surgical cancer patient have demonstrated the effectiveness of preoperative exercise training on HRQoL. ${ }^{39-41}$ Two recent systematic reviews in people with cancer undergoing adjuvant or neoadjuvant cancer treatment and surgery demonstrated that exercise training is safe and feasible and improves the physical fitness and HRQoL. ${ }^{42,43}$ Strengths of this study include that the evaluation of disability and HRQoL were performed by previously used methods for postoperative cancer patients such as modified Katz index and EuroQoL-5D-3L, respectively. ${ }^{3,8}$ The present study has several shortcomings. First, ADL and HRQoL were not compared before and after surgery. Second, although data were extracted from a prospective database, given its retrospective nature, selection bias may have influenced our findings. Finally, the sample size could be another limitation.

\section{Conclusion}

A little limitation for ADL is observed among patients undergoing thoracic or gastrointestinal surgery for cancer; however, advanced cancer, ASA class $\geq 3$ and postoperative major complications are risk factors associated with disability. With regard to HRQoL, mobility, pain/ discomfort and anxiety/depression are the most common affected dimensions. Advanced cancer, postoperative major complications and disability are risk factors associated with severe impairment in HRQoL. Recognizing these risk factors could aid in establishing a directed rehabilitation program for preventing disability and improve HRQoL in this population. Other studies confirming our results are required.

\section{Source of support}

Nil.

\section{Acknowledgements}

None.

\section{Conflict of interest}

The author declares no conflicts of interest.

\section{References}

1. Dirección de registros médicos y estadísticas de salud. Anuario Estadístico de Salud 2013. La Habana, Ministerio de Salud Pública de Cuba, cuba; Springer; 2014.

2. Zegers M, de Bruijne MC, de Keizer B, et al. The incidence, rootcauses, and outcomes of adverse events in surgical units: implication for potential prevention strategies. Patient Saf Surg. 2011;5:13.

3. Amemiya T, Oda $\mathrm{K}$, Ando $\mathrm{M}$, et al. Activities of daily living and quality of life of elderly patients after elective surgery for gastric and colorectal cancers. Ann Surg. 2007;246(2):222-228.

4. Aziz O, Atalla H, Lo B, et al. Ear-worn body sensor network device: an objective tool for functional postoperative home recovery monitoring. $J$ Am Med Inform Assoc. 2011;18(2):156-159.

5. Cevenini E, Invidia L, Lescai F, et al. Human models of aging and longevity. Expert Opin Biol Ther. 2010;8(9):1393-1405.

6. McAuley E, Szabo A, Gothe N, et al. Self-efficacy: Implications for Physical Activity, Function, and Functional Limitations in Older Adults. Am J Lifestyle Med. 2011;5(4)

7. Ulander K, Jeppsson B, Grahn G. Quality of life and independence in activities of daily living preoperatively and at follow-up in patients with colorectal cancer. Support Care Cancer. 1997;5(5):402-409.

8. Brown SR, Mathew R, Keding A, et al. The impact of postoperative complications on long-term quality of life after curative colorectal cancer surgery. Ann Surg. 2014;259(5):916-923.

9. Santos RF, Ribeiro JC, de Moraes FB, et al. Evaluation of the quality of life after vertebroplasty to treat compressive osteoporotic fractures. Rev Bras Ortop. 0214;49(5):477-481.

10. Young CJ, De Loyde KJ, Young JM, et al. Improving quality of life for people with incurable large-bowel obstruction: randomized control trial of colonic stent insertion. Dis Colon Rectum. 2015;58(9):838-849.

11. Lim HS, Cho GS, Park YH, et al. Comparison of quality of life and nutritional status in gastric cancer patients undergoing gastrectomies. Clin Nutr Res. 2015;4(3):153-159.

12. Ribeiro IL, Medeiros JJ, Rodrigues LV, et al. Factors associated with lip and oral cavity cancer. Rev Bras Epidemiol. 2015;18(3):618-629.

13. Zhou Q, Li K, Lin GZ, et al. Incidence trends and age distribution of colorectal cancer by subsite in Guangzhou, 2000-2011. Chin J Cancer. 2015;34(8):358-364.

14. Lykke J, Jess $P$, Roikjaer $\mathrm{O}$, et al. Increased lymph node yield is associated with improved survival in rectal cancer irrespective of neoadjuvant treatment: results from a national cohort study. Dis Colon Rectum. 2015;58(9):823-830

15. Homan J, Bökkerink GM, Aarts MJ, et al. Variation in circumferential resection margin: Reporting and involvement in the South-Netherlands. Eur J Surg Oncol. 2015;41(11):1485-1492.

16. Martos Benítez FD, Guzmán Breff BI, et al. Postoperative complications in thoracic and abdominal surgery: definitions, epidemiology and severity. Rev Cub Cir. 2016;55(1):40-53. 
17. Chen W, Fang Y, Mao F, et al. Assessment of disability among the elderly in xiamen of china: a representative sample survey of 14,292 older adults. PLoS ONE. 2015;10(6):e0131014.

18. Khoei MA, Akbari ME, Sharifi F, et al. Translation and validation of the activities of daily living scale with iranian elderly cancer patients treated in an oncology unit. Asian Pacific J Cancer Prev. 2009;14(5):2731-2737.

19. Alexandre TS, Corona LP, Nunes DP, et al. Gender differences in incidence and determinants of disability in activities of daily living among elderly individuals: SABE study. Arch Gerontol Geriatr. 2012;55(2):431-437.

20. Federman AD, Penrod JD, Livote E, et al. Development of and recovery from difficulty with activities of daily living: an analysis of national data. J Aging Health. 2010;22(8):1081-1098.

21. McLaughlin SJ, Connell CM, Heeringa SG, et al. Successful aging in the United States: prevalence estimates from a national sample of older adults. J Gerontol B Psychol Sci Soc Sci. 2010;65(2):216-226.

22. Janssen MF, Simon Pickard A, Golicki D, et al. Measurement properties of the EQ-5D-5L compared to the EQ-5D-3L across eight patient groups: a multi-country study. Qual Life Res. 2013;22(7):1717-1727.

23. Kimman M, Vathesatogkit P, Woodward M, et al. Validity of the Thai EQ-5D in an occupational population in Thailand. Qual Life Res. 2013;22(6):1499-1506.

24. Pattanaphesaj J, Thavorncharoensap M. Measurement properties of the EQ-5D-5L compared to EQ-5D-3L in the Thai diabetes patients. Health Qual Life Outcomes. 2015;13:14.

25. Chen PC, Kuo RNC, Lai CK, et al. The relationship between smoking status and health-related quality of life among smokers who participated in a 1-year smoking cessation programme in Taiwan: a cohort study using the EQ-5D. BMJ Open. 2015;5(5):e007249.

26. Obradovic M, Lal A, Liedgens $H$. Validity and responsiveness of EuroQol-5 dimension (EQ-5D) versus Short Form-6 dimension (SF-6D) questionnaire in chronic pain. Health Qual Life Outcomes. 2013;11:110.

27. Golicki D, Niewada M, Buczek J, et al. Validity of EQ-5D-5L in stroke. Qual Life Res. 2015;24(4):845-850.

28. Kim SH, Kim HJ, Lee SI, et al. Comparing the psychometric properties of the EQ-5D-3L and EQ-5D-5L in cancer patients in Korea. Qual Life Res. 2012;21(6):1065-1073.

29. Pickard AS, De Leon MC, Kohlmann T, et al. Psychometric comparison of the standard EQ-5D to a 5 level version in cancer patients. Med Care. 2007;45(3):259-263

30. Luo N, Li M, Chevalier J, et al. A comparison of the scaling properties of the English, Spanish, French, and Chinese EQ-5D descriptive systems. Qual Life Res. 2013;22(8):2237-2243.
31. Hoogerduijn JG, Schuurmans MJ, Korevaar JC, et al. Identification of older hospitalised patients at risk for functional decline, a study to compare the predictive values of three screening instruments. J Clin Nurs. 2010;19(9-10):1219-1225.

32. Khoei MA, Akbari ME, Sharifi F, et al. Translation and Validation of the Activities of Daily Living Scale with Iranian Elderly Cancer Patients Treated in an Oncology Unit. Asian Pacific J Cancer Prev. 2013;14(5):2731-2737.

33. West MA, Dimitrov BD, Moyses HE, et al. Timing of surgery following neoadjuvant chemoradiotherapy in locally advanced rectal cancerAcomparison of magnetic resonance imaging at two time points and histopathological responses. Eur J Surg Oncol. 2016;42(9):1350-1358.

34. Yang M, Ding X, Dong B. The measurement of disability in the elderly: a systematic review of self-reported questionnaires. $J$ Am Med Dir Assoc. 2014;15(2):150.

35. Buurman BM, Hoogerduijn JG, de Haan RJ, et al. Geriatric conditions in acutely hospitalized older patients: prevalence and one-year survival and functional decline. PLOS ONE. 2011;6(11):e26951.

36. Kawaguchi Y, Hanaoka J, Oshio Y, et al. Decrease in performance status after lobectomy mean poor prognosis in elderly lung cancer patients. $J$ Thorac Dis. 2017;9(6):1525-1530.

37. Saraiva MD, Karnakis T, Gil-Junior LA, et al. Functional status is a predictor of postoperative complications after cancer surgery in the very old. Ann Surg Oncol. 2007;24(5):1159-1164.

38. Makhabah DN, Martino F, Ambrosino N. Peri-operative physiotherapy. Multidiscip Respir Med. 2013;8(1):4.

39. Crandall K, Maguire R, Campbell A, et al. Exercise intervention for patients surgically treated for non-small cell lung cancer (NSCLC): a systematic review. Surg Oncol. 2014;23(1):17-30.

40. Singh F, Newton RU, Galvão DA, et al. A systematic review of presurgical exercise intervention studies with cancer patients. Surg Oncol. 2013;22(2):92-104.

41. Boereboom C, Doleman B, Lund JN, et al. Systematic review of preoperative exercise in colorectal cancer patients. Tech Coloproctol. 2016;20(2):81-89.

42. Loughney L, West MA, Kemp GJ, et al. Exercise intervention in people with cancer undergoing adjuvant cancer treatment following surgery: a systematic review. Eur J Surg Oncol. 2015;41(12):1590-1602.

43. Loughney L, West MA, Kemp GJ, Exercise intervention in people with cancer undergoing neoadjuvant cancer treatment and surgery: a systematic review. Eur J Surg Oncol. 2016;42(1):28-38. 